\title{
¿Es el cansancio de la técnica un factor a tener en cuenta en las infecciones peritoneales?
}

\author{
Paqui Gruart Armangué, Alex Andújar Asensio, Nieves Simal Velez, Esther Salillas Adot, Maricel Julve \\ Ibañez
}

Enfermeros/as. Unidad de DP. Hosp.U. de Bellvitge.CSUB. Hospitalet de Llobregat. Barcelona

\section{Resumen}

La infección peritoneal es una inflamación de la membrana peritoneal ocasionada habitualmente por una infección bacteriana.

Los efectos que genera una infección peritoneal son de vital importancia para el mantenimiento en el tratamiento de diálisis peritoneal. En algunas de estas infecciones se hace necesaria la retirada del catéter y consecuentemente el cambio a la Hemodiálisis cuya trasferencia a ella representa la quinta parte de las infecciones peritoneales.

Los motivos del fallo en la técnica dialítica son diversos.

El objetivo de nuestro estudio es revisar los factores que han podido influir en la incidencia de la infección peritoneal.

Se resalta que el $58 \%$ son Gram positivos, lo que significa que más de la mitad de los agentes causantes son microorganismos saprófitos de la piel, el 38\% son Gram negativos cuya posible causa es también la falta de higiene. El $4 \%$ es cultivo negativo.

Se comparan el tiempo de exposición con la de los episodios de infección peritoneal.

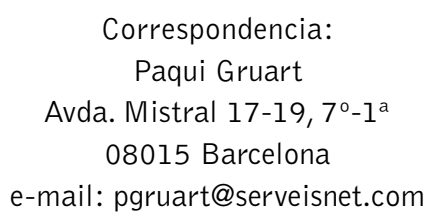

El personal destinado en la unidad de diálisis peritoneal en los años estudiados fueron 2 enfermeras/os de turno de 12 horas a días alternos y un enfermero de 15 horas semanales. En el 2009 la dotación se reforzó con una enfermera asistencial en turno de 7 horas mañana. Desde ese momento los programas de aprendizaje se hacen ininterrumpidamente por una misma enfermera en el turno que se pacta con el paciente.

Una mejor dedicación en el entrenamiento y la realización de reentrenos, disminuye la tasa de infección peritoneal ya que se puede detectar el cansancio y aburrimiento del paciente, antes de que aparezca una infección peritoneal.

\section{PALABRAS CLAVE:}

- DIÁLISIS PERITONEAL

- INFECCIÓN PERITONEAL

- REENTRENO

- CANSANCIO EN LA TÉCNICA

Is tiredness a factor to be taken into account in peritoneal infections?

\section{Abstract:}

Peritoneal infection is an inflammation of the peritoneal membrane usually caused by bacterial infection.

The effects generated by a peritoneal infection are of vital importance for maintaining peritoneal dialysis 
treatment. In some of these infections, it is necessary to remove the catheter and consequently change to haemodialysis, the transfer to which represents one fifth of peritoneal infections.

The causes of the failure in the dialytic technique are diverse.

The aim of our study is to review the factors that may have had an influence on the incidence of peritoneal infection.

It is noted that $58 \%$ are Gram-positive, which means that more than half of the causing agents are saprophyte micro-organisms of the skin, 38\% are Gram-negative, the possible cause of which is lack of hygiene. $4 \%$ are negative culture.

The time of exposure is compared to the episodes of peritoneal infection.

The staff allocated to the peritoneal dialysis unit in the years studied were 2 nurses on 12-hour shifts on alternate days and a nurse who worked 15 hours per week. In 2009 staffing was reinforced with a care nurse working a 7-hour morning shift. Since then learning programmes have been carried out without interruption by the same nurse in the shift agreed with the patient.

Better dedication to training and carryout out retraining reduces the rate of peritoneal infection as patient tiredness and boredom can be detected before a peritoneal infection appears.

\section{KEY WORDS:}

- PERITONEAL DIALYSIS

- PERITONEAL INFECTION

- RE-TRAINING

- TIREDNESS OF THE TECHNIQUE

\section{Introducción}

La infección peritoneal es una inflamación de la membrana peritoneal ocasionada habitualmente por una infección bacteriana.
La puerta de entrada más frecuente de los gérmenes está relacionada con el catéter y la vía intraluminal, por donde entran las bacterias saprofitas de la propia piel. Durante el aprendizaje de la técnica se le enseña al paciente el lavado de manos, secado completo con toalla de un solo uso y antes de las conexiones/desconexiones, frotado de manos con líquido antiséptico, con el fin de poner las barreras necesarias para evitar esta vía de entrada de los gérmenes.

La membrana peritoneal puede quedar alterada por una infección persistente y agresiva además de que el paciente padece anomalías metabólicas, como aumento de pérdida de proteínas, pérdida de ultrafiltración y los signos propios de una infección como dolor abdominal, malestar general y posiblemente fiebre, siendo esta, una de las causas mayores del ingreso hospitalario y del deterioro del propio paciente.

Los efectos que genera una infección peritoneal son de vital importancia para el mantenimiento en el tratamiento de diálisis peritoneal. En algunas de estas infecciones se hace necesaria la retirada del catéter para su curación y consecuentemente el cambio a otro tratamiento sustitutivo renal que en este caso será la HD. El fallo de la técnica dialítica y la transferencia a Hemodiálisis representa la quinta parte de las infecciones peritoneales ${ }^{1}$.

Los motivos del fallo en la técnica dialítica son diversos. Aunque no esté demostrado, el estado depresivo y la falta de interés en la técnica de los pacientes podría ser una de las causas de la peritonitis ${ }^{2}$. El cansancio en la técnica podría ser también otro de los motivos para dejar de cumplir con la técnica dialítica enseñada. Por esta razón se hacen necesarias las visitas domiciliarias y el reciclaje de los pacientes, para poder entender su estado de ánimo, aclarar dudas y proporcionar seguridad al paciente $y / 0$ cuidador 3 .

Según la Sociedad Internacional de Diálisis Peritoneal, "las enfermeras de DP con excesivo número de pacientes tendrán dificultades para poder enseñar correctamente al paciente y no habrá el suficiente tiempo para el reentreno cuando el paciente lo necesite ${ }^{4 \prime \prime}$.

En nuestra unidad durante los tres últimos años ha habido varios cambios, en el personal de enfermería, aumento de trabajo en la unidad, disminución del tiempo para la dedicación que precisa cada paciente y en consecuencia han surgido diferentes complicaciones, 
sobre todo infecciones peritoneales con distinta incidencia en estos tres años.

Por este motivo, el objetivo de nuestro estudio es revisar los factores que han podido influir en la incidencia de la infección peritoneal.

\section{Población y Método}

Estudio retrospectivo descriptivo de tres años, desde enero del 2007 a diciembre del 2009 revisando sexo, edad a la colocación del catéter, tiempo de exposición. También se ha estudiado el número de aprendizajes para la DP hecho por las enfermeras de la unidad y el ratio de trabajo por enfermera. Comparándose todo ello con los episodios de infección peritoneal que han sufrido nuestros pacientes en el tiempo descrito.

El tratamiento estadístico se ha hecho con el paquete Stat View y SPSS13.0.

\section{Resultados}

Durante los tres años estudiados, se han tratado 191 pacientes entre los prevalentes e incidentes. En la tabla 1 se puede contemplar las edades, sexo y $n^{\circ}$ de infecciones peritoneales, dispuestas según sexo.

\begin{tabular}{|c|c|c|c|c|}
\hline $\mathbf{n}$ & Edad & Tiempo exposición & n peritonitis \\
\hline 57 & $56.12 \pm 15.08$ & $25.57 \pm 20.88$ & $17(29.82 \%)$ \\
\hline 134 & $55.73 \pm 13.08$ & $24.06 \pm 18.05$ & $32(23.88 \%)$ \\
\hline
\end{tabular}

Tabla 1. Distribución de pacientes y $n^{0}$ de infecciones peritoneales.

En la distribución por gérmenes (Figura 1 ), se resalta que el $58 \%$ son Gram positivos, lo que significa que más de la mitad de los agentes causantes son gérmenes saprofitos de la piel, del agua y/o de mala práctica en la técnica. El $38 \%$ son Gram negativos cuya posible causa sea también la falta de higiene o secado incorrecto de las manos. El $4 \%$ es cultivo negativo.

En el desglose por años se puede observar que el $n^{\circ}$ de pacientes atendidos no varía mucho (Figura 2 ) entre los distintos años, pero la incidencia de infección peritoneal es muy diferente, siendo en el 2007 la incidencia de 26,04 meses/paciente y de 24,07 y 69,54 en el 2008 y 2009 respectivamente.

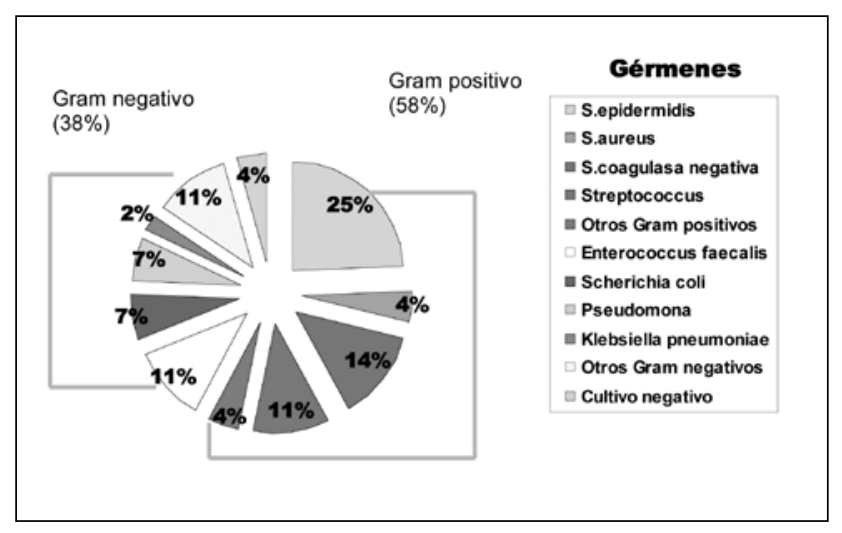

Figura 1. Gérmenes encontrados en los cultivos de líquido peritoneal

En las Figuras 3 y 4 se describe la edad y el tiempo de exposición, comparándolos con la incidencia de infección peritoneal. Cabe destacar que en el año 2008, los pacientes padecen una infección peritoneal, con menos tiempo de exposición. Entendemos por

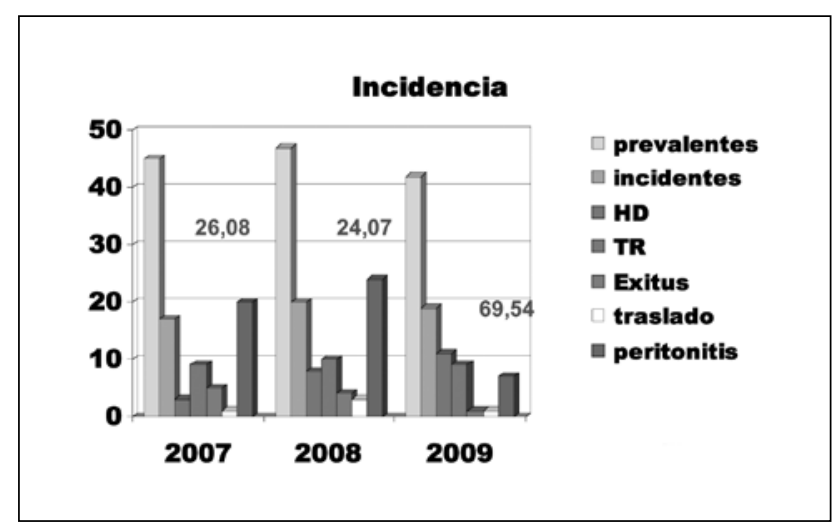

Figura 2. Incidencia de infección peritoneal

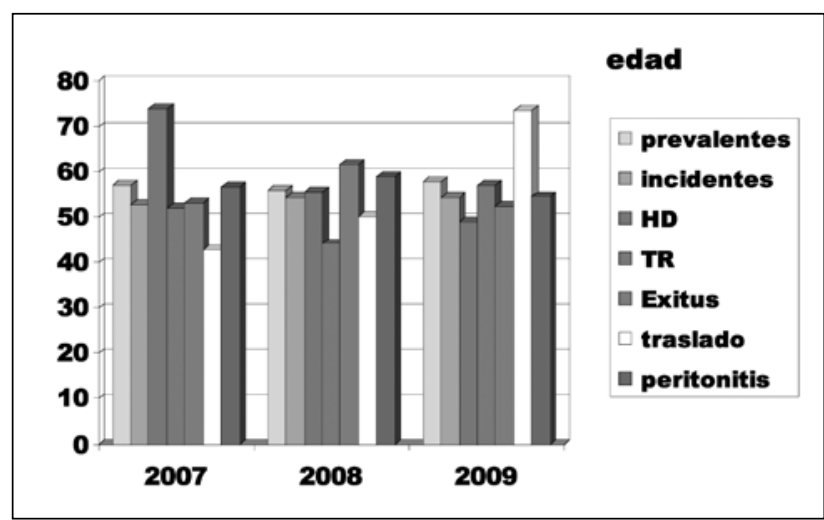

Figura 3. Edad media de los pacientes al final de DP 


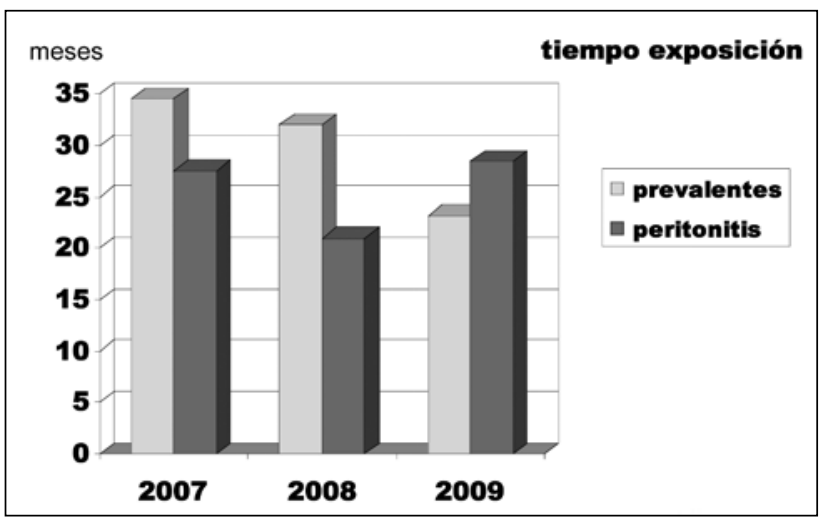

Figura 4. Tiempo de exposición vs infección peritoneal

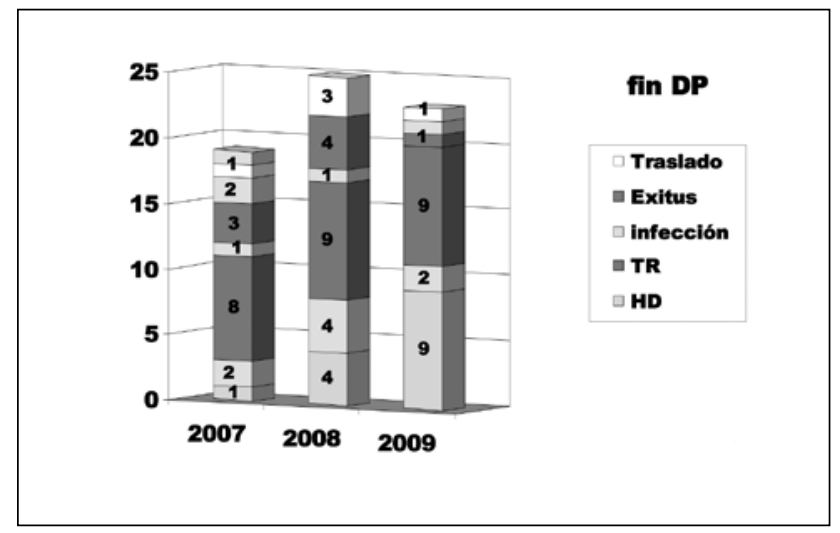

Figura 5. Distribución de las infecciones peritoneales vs fin de dialisis peritoneal

tiempo de exposición al tiempo que el paciente lleva desde la fecha de colocación del catéter peritoneal. Al comparar el tiempo de exposición (Figura 5) con la de los episodios de infección peritoneal, no encontramos diferencia significativa puesto que existen grandes desviaciones en las medias y el tiempo de exposición dista mucho entre la máxima y la minima. No obstante, cabe destacar que el $11,11 \%, 16 \%$ y $9,1 \%$ pasan a HD como consecuencia de una infección peritoneal, ya sea por recidiva o imposibilidad de seguir con la DP por deterioro de sus patologías concomitantes.

En cuanto al ratio de trabajo realizado por enfermería de DP ha sido, además del de asistencia al paciente hospitalizado, visitas programadas y de urgencias, cambios de prolongador, $\mathrm{PEP}, \mathrm{y}$ todos los actos propios de la enfermera de DP, los aprendizajes hechos en los años descritos pueden contemplarse en la Tabla 2. Cabe destacar que en el año 2008, año de acontecimientos, hubo más pacientes incidentes.

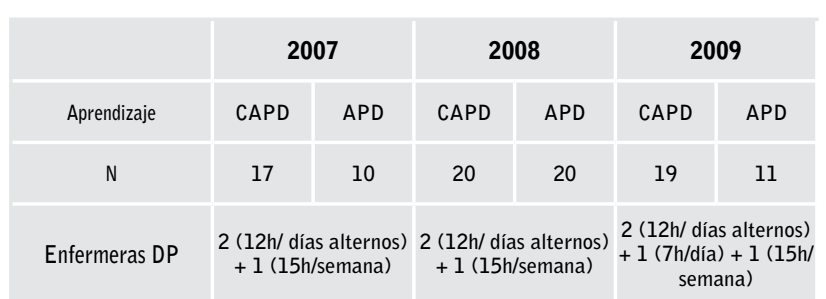

Tabla 2. Aprendizajes vs personal disponible en la unidad.

El personal destinado en la unidad de diálisis peritoneal en los años 2007 y 2008 fueron 2 enfermeras/os de turno de 12 horas a días alternos y un enfermero de 15 horas semanales.

Al haber un único responsable, los programas de aprendizaje eran interrumpidos por las distintas actividades del servicio (visitas, curas, revisiones, hospitalizados, pruebas complementarias, asistencia a la colocación de catéteres, etc.). En el año 2009, la dotación se reforzó con una enfermera asistencial en turno de 7 horas mañana. Desde ese momento los programas de aprendizaje se hacen ininterrumpidamente por una misma enfermera en el turno que se pacta con el paciente.

No se han hecho visitas domiciliarias rutinarias, durante este tiempo, ni reciclajes. Únicamente cuando el paciente acudía a la unidad de visita y/o urgencias, según el estado en que se hallaba y si era posible, se revisaba la técnica y se le recordaban los puntos más frágiles.

\section{Discusión y conclusiones}

Aunque estadísticamente no hemos encontrado diferencias significativas, los resultados son visibles. Pensamos que el corte del año 2007 al 2009 ha sido casual, solo para comparar un año, el 2007, sin mayores problemas, contrastándolo con el 2008 en el que hubo muchos acontecimientos, y el del 2009, un año de cambios.

Los microorganismos causantes de la infección peritoneal, nos indican que la preparación y el lavado de manos está fallando ya que casi todos los gérmenes culpables de la infección peritoneal se pueden encontrar en las manos, por mala higiene o transportadas por el agua contaminada, por falta de secado correcto de las manos y/o fallo en la antisepsia. Varias de 
las bibliografías consultadas, demuestran que en la revisión de la técnica, mas del $25 \%$ de los pacientes revisados no se lavan correctamente las manos y el desinfectante está mal aplicado o a destiempo. Pensamos que a pesar de no poder hacer las visitas domiciliarias ni reciclajes, por infraestructura del servicio, deberemos buscar un sistema en el que podamos prevenir y hacer reciclaje en la técnica, cuando detectemos la necesidad.

Como dice Troidle y colaboradores ${ }^{2}$ que los estados depresivos pueden ser causa de la inflamación peritoneal, creemos que la falta de interés también puede ser causa de ello ya que a pesar de que no hay diferencia significativa estadísticamente, hay pacientes que pasado algún tiempo del inicio de la DP y, al comprobar que no ocurre nada, bajan las defensas a la infección instruidas durante el aprendizaje y sufren una infección peritoneal. Esto podría ser una de las bases en la que se fundamenta la necesidad de la visita domiciliaria y del reciclaje ${ }^{3}$, ya que mantendría en alerta al paciente y la enfermera visitadora de DP podría detectar el momento en el que se inicia el proceso de depresión y la posible decadencia en la técnica pero solo se deben hacer las visitas domiciliarias si las enfermeras de DP tienen el suficiente tiempo para hacerlas ${ }^{2}$.

Como afirma el Comité de la diálisis peritoneal internacional $^{4}$, la sobrecarga de las enfermeras con excesivo número de pacientes tiene poco tiempo para el entrenamiento y no dispone del suficiente para hacer los reentrenos necesarios, como nos ha ocurrido a nosotros, ya que los microorganismos causantes, en gran numero, han sido por fallo o disminución de la higiene de manos o de la asepsia. La duración del entrenamiento debe ser el adecuado para cada paciente y si no se dispone de él, el cansancio y aburrimiento hace que no se ponga interés en lo que se explica y lo que se aprende. Según afirma la bibliografía, una mayor duración en el entrenamiento, si es necesaria y la realización de reentrenos disminuye la tasa de infección peritoneal ${ }^{1}$, el tiempo del que no hemos dispuesto en nuestra unidad.
Por los resultados antes expuestos, podemos afirmar que el cansancio en la técnica y la falta de motivación del paciente, ya sea por fallo de estímulo por parte del personal o del propio paciente, influye en el desarrollo de episodios de infección peritoneal.

Recibido: Marzo 2011

Revisado: Abril 2011

Modificado: Mayo 2011

Aceptado: Julio 2011

\section{Bibliografía}

1. Montenegro. J, Molina. A, Rodriguez-Palomares, J.R. Gonzalez-Parra, E. Peritonitis en DP. En: Guías de práctica clínica en Diálisis Peritoneal. Guías SEN. 2006. Vol 26, sppl 4: 115-131.

2. Troidle L, Sorban-Brenan N, Kliger A, Filkestein F: Continuous peritoneal dialysis-associated peritonitis: a review and current concepts. Seminars in Diálisis, 2003.16 (6): 424-427.

3. Gruart, P. Arias, N. Reentrenamiento en DP. En: Coronel, F. Montenegro, J. Selgas, R. Celadilla, 0 . Tejuca, M. Manual práctico de Diálisis Peritoneal. $2^{a}$ ed. SEDEN-SEN. Atrium Comunicación Estratégica S.L. 2005. 44: 257- 362.

4. PD Related infections. Recomendations PDI. March, 2005. Vol 25, n 2.

5. Bajo, MA. Selgas, R y cols. Indicadores de infecciones. En: Plan de Calidad científico técnico y de mejora continua de calidad en DP. Sociedad Española de Nefrología (Grupo de Promoción del Conocimiento en DP), 2007. 7. 